\title{
Tensions between Individual Use and Network Adoption of Social Media Platforms
}

\author{
Nikhil Srinivasan and Jan Damsgaard \\ Department of IT Management, Copenhagen Business School, Frederiksberg, Denmark \\ \{ns.itm, jd.itm\}@cbs.dk
}

\begin{abstract}
Social media have diffused into the everyday lives of many but still pose challenges to individuals regarding use of these platforms. This paper explores the multiple manners in which social media platforms gets employed by individuals based on an examination of 4 vignettes generated by interviewing individuals within a university context. An analysis of the vignettes and individual use behaviors highlights the tension between network-based adoption of social media platforms and the constraints that the network places on individual use of the platform.
\end{abstract}

Keywords: social media, adoption, use, social network, tensions.

\section{Introduction}

Social media platforms have in a very short time integrated well into people's lives, both socially and professionally. Long-lost school mates reconnect with one another, friends and family share news, and professional bonds are easily continued after a work relation has ended; all as a result of social media platforms. A social media platform is built around social relations that individuals establish or confirm with each other through information and communication technologies for the purpose of communication, collaboration and coordination of information, knowledge and activities. A social media platform is inherently a participative environment where the consumers of information and knowledge are simultaneously the co-creators and consumers of new information and knowledge, while at the same time interacting with and engaging with individuals (Parameswaran et al. 2007).

Academic studies have examined the diffusion and adoption of specific social media technologies (Hester 2008; Hester et al. 2008) and employed popular and widely acknowledged models such as Diffusion of Innovation (DOI) (Rogers 1995), Technology Acceptance (TAM) or Unified Theory of Acceptance and Use of Technology (UTAUT) (Davis et al. 1989; Venkatesh et al. 2003) that have proven themselves in the past as useful vehicles in explaining the diffusion and adoption of IT in general. However extensions of DOI and TAM to cover social media platforms do so without revisiting the basic assumptions of the models and their validity domain (Sledgianowski et al. 2009; Tufekci 2008). This results in a conflation of individual use behaviors on the part of the individual with social network adoption contexts. 
This conflation of individual use and network adoption is significant because the one profound difference between general IT and social media platforms is that social media platforms are communal and have inherent network effects. Social media platforms only have value when many are using them, which is not paramount for many previous IT applications that were perfectly useful for the individual alone: e.g. wordprocessing. Consequently, examining social media platforms from the perspective of TAM and DOI tend to emphasize their individual use behaviors and the antecedents to those behaviors, rather than a broader examination of those individual use behaviors within a situated network (Granovetter 1973).

We contend that while DOI and TAM do shed light on examining adoption and diffusion of IT in general, social media represents a new paradigm in IT and as such traditional models of adoption and diffusion may not readily apply. The goal of this paper is to explore the adoption and diffusion of social media platforms. The central point of departure is the following research question.

\section{How does the networked nature of social media platforms influence their use by individuals?}

The remainder of this paper is organized the following way. In the next section we revisit classical adoption and diffusion theories in light of social media platforms. We then examine the nature of social media platforms and the characteristics that describe them. We then describe a field study designed to examine the tension between individual use and network adoption of social media platforms. We subsequently discuss our field study results and make some conclusions and note some limitations in our study.

\section{Revisiting Classical Adoption and Diffusion Theory}

This section discusses how the diffusion and adoption of technologies have been previously examined.

\subsection{Technology Adoption}

The individual adoption of a technology is based on two broad theories from social psychology i.e. the theory of reasoned action (Fishbein et al. 1975) and the theory of planned behavior (Ajzen 1985). The theories were immediately absorbed by information systems researchers and formed the basis for some of the most interesting, vibrant and comprehensive research programs of the discipline. The first work by Davis, Bagozzi and others $(1989$; 1989) was referred to as the technology acceptance model (TAM) and was subsequently built on by others to extend the model. The theory has been applied to compare across cultures (Straub et al. 1997) and genders (Gefen et al. 1997), extended with social influence (Malhotra et al. 2002), accounted for task-technology fit (Dishaw et al. 1999), and other similar extensions. A comprehensive examination of the theory of technology acceptance was performed by Venkatesh et al. (2003) who compared 8 different user acceptance models and synthesized them into a comprehensive model referred to as the Unified Theory of Acceptance 
and Use of Technology (UTAUT). Work still continues in this program of research with an examination of IS continuance (Bhattacherjee 2001) that examines the nature of continued use past the point of initial adoption and the role of habit (Limayem et al.). Since a complete review of the theory is beyond the scope of the paper please refer to Venkatesh (Venkatesh et al. 2007; Venkatesh et al. 2003) and the special issue of JAIS $^{1}$ for a more extensive review of the literature in this domain.

Broadly speaking within the individual technology acceptance literature, characteristics such as habit, self-efficacy, experience, task relevance, and others are primary and important determinants of behavior regarding individual technology adoption and use (Davis et al. 1989; Venkatesh et al. 2003). This perspective specifically privileges endogenous characteristics of the individual and individual motivations in ultimately determining behavior. Characteristics such as social norms and self-image are employed as mediating or moderating roles in influencing individual perceptions of the usefulness of a specific technology or service.

\subsection{Technology Diffusion}

The second perspective on the spread of technology exists at a broader level and examines the factors that contribute to the manner in which technologies diffuse across a population of potential adopters. This second perspective is often referred to as the diffusion of innovation (DOI) theory. While developed in the late $19^{\text {th }}$ century, it is only recently that IS researchers have started examining and applying the theory. The seminal work of Rogers (1995) examines a large body of work in this domain and synthesizes it. Since the work of Rogers, DOI theory has been extensively applied in the context of IT (Attewell 1991; McFarlan et al. ; Moore et al. 1991; MustonenOllila et al. 2003) to examine the diffusion of technological innovations and information technologies. Much of the information systems research literature has examined the adopter populations within organizations with a focus on the factors that influence adoption (Bala et al. 2007; Lin 2006; Melville et al. 2008). However despite its extensive application and popularity, DOI has also received some criticism (Lyytinen et al. 2001b; Lyytinen et al. 2011) noting that information technologies are often quite complex and that diffusion theories may not be sophisticated enough to address such complex technological innovations. DOI theory is especially effective at examining singular, monolithic technologies or well-defined systems with an apparent function such as TV sets or coffee makers (Lyytinen et al. 2001b). Such technologies typically rely on economies of scale on the supply side. The use of the system on the demand side is fairly independent of others' use of the same technology.

\subsection{Other Related Theories}

Social influence theories have also been applied to examine technology adoption among individuals with the basic premise that individual decisions and attitudes are not developed in isolation (Salancik et al. 1978). Individuals refer to a social context and environmental cues in order to aid in the decision making process. Social and

1 http://aisel.aisnet.org/jais/vol8/iss4/ 
structural cues are conveyed through the social relations that individuals share and behavioral grammars reflect the influencers' autonomy, consistency or rigidity (Kraut et al. 1998). Social influence and social information processing theories do lend insight into examining use and adoption behaviors of individuals; but while it explains certain types of use, it does not explain non-use by individuals that exist in similar social contexts.

Critical mass theories of information technology postulate that mass adoption and diffusion of technologies take place based on momentum built up by a social system. The momentum builds up based on social factors within a system such as size, interrelatedness of individuals and levels of communication. Critical mass theories also suggest that there is a point at which enough users have adopted an innovation or technology that the acceleration of adoption process is self-sustaining (Sledgianowski et al. 2009) which is also referred to as the "bandwagon effect" (Peng 2010). Critical mass theories have also been examined in the context of adoption of new telecommunication services (Mahler et al. 1999), online gaming (Hsu et al. 2004), contribution to public knowledge databases (Peddibhotla et al. 2007), adoption of EDI systems (Iacovou et al. 1995) and more. These studies highlight the self-sustaining effects that adoption behavior of certain technologies may have. Markus (1987) proposed a critical mass theory for interactive media where she suggests that examining individuals' technology adoption and use decisions may not be sufficient to predict behavior at the community level due to the interdependence in the use of certain technologies. This suggests that certain information and communication technologies need to have a unique lens applied to examine their adoption and diffusion processes.

\subsection{Revisiting the Assumptions of TAM and DOI}

In the TAM approach to the adoption of innovations and technologies, individual characteristics are the primary determinant of individual adoption and use behavior. The limitation of such a theory is that the individual is the primary unit of analysis without an examination of the embeddedness (Granovetter 1973) of the individual in the context of their social networks. The role of social networks and embeddedness in such theories takes the form of subjective norms through which influence operates (Vannoy et al. 2010). DOI theory has been found as ineffective when examining diffusion of complex, networked information technologies (Lyytinen et al. 2001b). In examining the adopter population, individuals are treated as relatively isolated from the group, thereby separating them from the social setting in which they are embedded. By treating individuals as independent in their adoption behavior, the TAM and DOI perspectives do not focus on the interplay between users and between user behaviors (Benbasat et al. 2007) that involve more than one system and might involve an infrastructure of systems. This is a specific neglect of group, social and cultural aspects of decision-making behavior and a lack of examination of self-regulation processes (Bagozzi 2007). Traditional TAM and DOI models are less suitable in situations where adoption decisions are joint processes of decision-making or "weintention" (Bagozzi 2007), that are rooted in a collective intention to adopt. Attention paid to the collective or "we-intention" examines the role of valence or feelings that 
individuals in a group have to each other and to choices of the group on the intention to adopt a technology (Sarker et al. 2005). "We-intention" or "we-ness" refers to the interdependence of individuals in making technology adoption decisions where relational linkages play a key role (Sarker et al. 2010).

Furthermore, TAM and associated theories of adoption do not adequately explain why parts of the population are more likely to adopt the technology or service and the other parts of the population less so, despite sharing similar individual characteristics (Lyytinen et al. 2001a). While they do a good job of explaining why specific individuals adopt technologies and service, they do a relatively poor job of understanding and explaining why others do not adopt a technology or service despite being similar and belonging to the same pool of potential adopters (Lyytinen et al. 2001a).

\section{The Nature of Social Media Platforms}

Social media platforms refer to technological and social infrastructures that are used to support specific and generalized modes of communication and collaboration between distributed individuals that share a common interest. They are also avenues for content generation where persons individually and collaboratively create, contribute and build on others' information contribution. Social media has been described as having the unique feature of "active creation of content by their users or members" (Scott et al. 2009) where the creation of content takes place through the building and maintaining of social networks and relations. Social media or social media platforms are collective, distributed and fluid, and as such are both complex and highly topical. While social media and social media technologies are easier to distinguish from traditional computer mediated communication environments, they are harder to distinguish from one another (Hanna et al. 2011). Social media technologies enhance communication effectiveness with customers and make them part of the organization's environment. The technologies extend the organization's antenna and moves it closer to the customer (Jones et al. 2009). The social media technologies described, and the organizations and social networks adopting them, are presented with several opportunities and challenges. Several strategies (Dutta 2010) have actively been developed for managing the social media technology environment ranging from personal to professional and private to public.

A honey comb structure (Figure 1) can be used to represent the various capabilities of social media and social media platforms (Kietzmann et al. 2011). The structure encapsulates seven functional capabilities that social media may have. These capabilities are presence, sharing, relationships, identity, conversations, groups and reputation. The identity component represents the extent to which users reveal their identities in the social media setting such as disclosing their name, age and other personal information. Conversations represent the extent and abilities of the system to enable users to communicate with other users in the social media and social media environment. Sharing represents the extent to which users exchange, distribute and receive content. Social media is based around the social interconnectedness between individ- 
uals and consequently sharing forms an important part of social media and social media environments. Presence represents the capability in the system to inform the users as to whether other users are accessible within the system. Identifying copresence in virtual environments such as social media and social media platforms the relationships components represents the extent to which individuals can relate to or establish relationships with other users. This represents the sociality component of social media. Reputation is the extent to which users can identify the standing of others in a social media and social media setting. Groups represent the extent to which users can form communities and subgroups within the social media and social media setting.

These 7 elements of the honeycomb structure represent characteristics and functionalities that are present within applications of the social media ecosystem. Various tools provide combinations of these functionalities to achieve their intended purpose. For instance, Facebook is based around the characteristic of relationships but goes on to provide functionality in the domains of identity, reputation, conversations and presence. Foursquare, another social media and social media tool is based around the notion of presence but also provides functionality around the notions of identity and relationships.

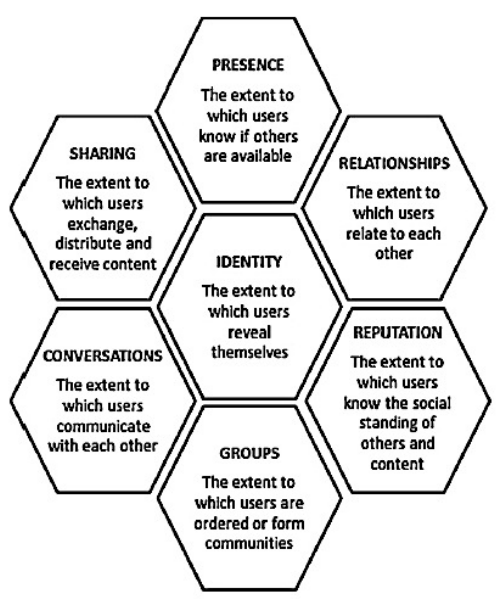

Fig. 1. Social Media Functionality

These 7 characteristics also highlight the Janus-faced nature (Arnold 2003) of social media platforms. Social media platforms provide individual use affordances through identity, reputation and presence functionality but enable network behaviors through sharing, relationship, conversation and grouping functionality.

The value to the users of social platforms is based on their appropriation of the functionalities based on individual use behavior or network appropriations based on number of their friends, family and colleagues they can communicate and collaborate with, within the specific social media platform. Thus while a single individual may be mobile across social media platforms, the social network and an individual's 
interactions with that network are locked into the specific platform used by the social network. Subsequently an individual's use of social media platforms is poised between individual use of functionalities and network use based on their communication and information needs with the relevant social networks.

\section{Differentiating Social Media Platforms from other Technologies}

Social media platforms are complex and are composed of disparate and fragmented networked systems that are the infrastructure on which social networks create content, communicate and collaborate. Individuals are not isolated on social media platforms. Architectures of participation and networks of users are fundamental to the notion of "collective intelligence" that form the basis of social media platforms (O'Reilly 2005). Social media platforms as infrastructures bind social networks together through which existing and new social relations are established and maintained. Network externalities result from the use of systems by friends, neighbors and families of individuals and consequently make social media platforms more valuable.

In addition to the network effects that are inherent in social media platforms; social networks add their own unique twist to technology adoption. Social networks are important in social media services since they are likely the first and major sources of influence in adoption decisions. The local configuration of relationships and the social network's adoption of technology represents a "we-intention" (Bagozzi 2007) on part of the social network. The migration of traditional social networks of friends, colleagues, family and acquaintances to digital networks has often resulted in the clash of these various networks. In addition to making the various social networks available on common technological platforms, these networks now are visible to each other. The availability and visibility of social networks however come at a price; the flattening or collapsing of the relationships into single homogeneous relationships, resulting in uncomfortable experiences with them (Boyd 2007). Furthermore, Boyd (2007) found that in the case of Friendster, the social network of individuals played a significant role in both the initial and second phases of adoption. This adoption was prior to the discussion in popular media, and invitation to use the service was based on whether your friends considered you a fit within the specific community that you were invited to. The observation that individuals belong to several social networks; e.g., professional, sports, relatives etc., suggests that social networks may adopt different social media platforms for supporting their social relations. This may mean that an individual may adopt several such platforms, essentially one for each network that they participate in or belong to. Thus the social network must form the key factor in the adoption of social media platforms, given the network effects associated with them.

The honeycomb framework that was described in figure 1 identifies several functional characteristics of social media platforms. These characteristics are centered on the affordances provided to the individual by social media platforms. While the affordances and functional capabilities of social media are individual centered, they are also relationship and network oriented. For instance, the functional capability of sharing which is the ability to exchange and distribute content is provided by the social media platform to the individual, but the exchanging and distribution of the content can either be one-to-one, one-to-many or one-to-all. Similarly, presence, a functional 
capability provided to the individual to communicate with and share presence information with others highlights the individual use nature of social media platforms while conversations highlights the social network oriented nature of social media platforms.

\section{$4 \quad$ Research Method}

Through a field study, we examine the central thesis in this paper, which is that social media platforms exist within a duality of individual use and social network adoption. The field study allows us to examine the context of adoption, and the adoption and use process of the social network's use of social media platforms (Lee 1989; Yin 1989).

\subsection{Field Study Design}

We employed semi-structured interviews as a data collection technique. The semi-structured interview started by focusing on the broad concept of the study and then narrowed down into specific instantiations of the adoption of social media by the interviewees. We started by probing into the use of social media and other communication technologies by the individual. We then asked the individual to describe the specific technologies in detail with emphasis on the social media technologies that specific groups and cliques employ in the interviewee's network. As such we asked for an individual interviewee to speak on the various social networks he or she may be participating in and his or her adoption and use of the social media platform for these networks.

\subsection{Data Collection}

This field study was conducted in a university setting. This setting is appropriate for data collection purposes as such populations are likely to use the social media services and are more amenable to having their behavior and use. The informants employ a variety of communication technologies and of course belong to a number of social networks. We probed the informant about the communication technologies their social networks employed, and for each social network that the person named we asked:

1. The genesis, name, size and history of the social network

2. The social network's general use of social media platforms and other communication technologies

3. The date of initiation and the adoption among the members of the social network

4. The number of close friends, followers and relationships the social network has. 
5. If there is a core of strong ties that forms the network, and what weak ties are common

6. How the members of the social network previously communicated.

The interviews lasted between 30 to 45 minutes. The interviewees were recruited from a marketing class. Students were invited to participate in the study by one of the authors.

\subsection{Data Analysis}

We employ vignettes (Avital et al. 2009; Jarvenpaa et al. 2000) to describe the adoption and use of social media platforms by social networks and individuals. We start by discussing the data analysis process and the manner by which various vignettes were constructed. We then proceed to discuss the vignettes that were elicited.

\section{Method}

Vignettes are used to illustrate the complex use of social media platforms. Our analysis of the observed social networks has been summarized in four vignettes. The interview subjects themselves did not provide the vignettes that are described. These vignettes were constructed by the authors from the narratives described by the interviewees and are used to illustrate the Janus-faced nature of social media platforms.

The vignettes were identified based on questions discussed previously. The interviewees were asked to respond to the questions and upon identification of a specific network, were asked to detail specific characteristics of the network and the communication tool used therein. When the interviewee provided a social network that employed the social media platform as a tool, they were asked about the unique characteristic or feature of the tool they employed for that social network and the reason why such a feature was employed. This enquiry process across all the interviews provided opportunities for the interviewees to reflect on their unique experience as it relates to social media. It also allowed researchers to focus on both the overlapping and unique characteristics of social media platform use. Interviewees identified several overlapping vignettes and a few unique ones. The vignettes subsequently represent the various manners of use of the social media platform by the interviewees. We use the vignettes as short illustrations of these use scenarios. These vignettes are summarized in Table 1.

Prior to the actual discussion of communication and social media technology use, the interviewees were asked to describe their daily use of the Internet and other IT. All interviewees were very accomplished with respect to communication and social media technologies. Most if not all of them spent several hours a day on the Internet. The time they spent on the Internet was a combination of school activities, job activities and hedonic activities. They attempted to articulate the amount of time spent on each of these activities but were cognizant of the fact that the manner in which they allocate time to each of these specific activities was fluid. They switched 
Table 1. Characteristics of the four vignettes

\begin{tabular}{|c|c|c|c|c|}
\hline Vignette & Social network & Representative & $\begin{array}{l}\text { Social media } \\
\text { platform }\end{array}$ & Frequency \\
\hline $\begin{array}{l}\text { As a Commu- } \\
\text { nication and } \\
\text { Coordination } \\
\text { Medium }\end{array}$ & $\begin{array}{l}\text { Jewish Summer } \\
\text { Camp, High } \\
\text { school cohort } \\
\text { and Soccer } \\
\text { Team }\end{array}$ & Udi Jannik & Facebook & $\begin{array}{l}\text { A few } \\
\text { times a } \\
\text { week }\end{array}$ \\
\hline $\begin{array}{l}\text { As a Relational } \\
\text { Continuance } \\
\text { Device }\end{array}$ & $\begin{array}{l}\text { University Co- } \\
\text { hort }\end{array}$ & Shu & Facebook & $\begin{array}{l}\text { A few } \\
\text { times a } \\
\text { week }\end{array}$ \\
\hline $\begin{array}{l}\text { As an Observa- } \\
\text { tional Platform }\end{array}$ & $\begin{array}{l}\text { Squash Team, } \\
\text { College Net- } \\
\text { work Profes- } \\
\text { sional Network }\end{array}$ & $\begin{array}{l}\text { Casper Udi } \\
\text { Khaled }\end{array}$ & $\begin{array}{l}\text { Facebook } \\
\text { LinkedIn }\end{array}$ & $\begin{array}{l}\text { A few } \\
\text { times a } \\
\text { month } \\
\text { Once a } \\
\text { week }\end{array}$ \\
\hline $\begin{array}{l}\text { As Emerging } \\
\text { Social Infra- } \\
\text { structure }\end{array}$ & $\begin{array}{l}\text { Family and } \\
\text { friends }\end{array}$ & $\begin{array}{l}\text { Casper Khaled } \\
\text { Shu }\end{array}$ & $\begin{array}{l}\text { Facebook, } \\
\text { LinkedIn }\end{array}$ & $\begin{array}{l}\text { A few } \\
\text { times a } \\
\text { month }\end{array}$ \\
\hline
\end{tabular}

seamlessly between the activities. The interviewees were highly fluent in the use of computer and communication technologies such as phone calls, e-mail, SMS, internet chat, Voice over IP calls and social media environments such as Facebook, Twitter, LinkedIn and others. They often employed more than one form of communication technology and were able to articulate the communication and social media technologies effectively. Most of them spent about $25-50 \%$ of their daily time on the Internet on communicative activities.

\section{Vignette 1: Social media is a Communication and Coordination Medium}

This vignette focuses on one specific social network that was formed during participation in a Jewish summer camp and it illuminates how a social network uses a social media platform as a communication and coordination medium. The social network is made up of people that were present at the camp during the same summer. After the camp, communication in the social network was through e-mail threads used to sustain conversation or initiate new communication. Any new communication would employ previous threads to start the communicative or coordinative activity. This email thread switched over to a Facebook group in 2006. When the core of the camp network moved to Facebook, the remainder of the cohort were de facto forced to drop the use of e-mail chains to communicate and started employing the Facebook Social media platforms to communicate in the social network. Furthermore new camp networks form their own social networks within the Facebook platform and employ the platform to communicate and coordinate their activities. These activities may include future summer camp participation, visits to Israel, parties and events, travels abroad, 
etc. In addition to the network of the summer camp, high school cohort and soccer club networks also communicate through the Facebook Social media platform.

While the familial and friend networks of the informants may employ the more traditional modes of communication, other networks employ social media based platforms. For instance, squash-based social networks rely on weak ties to organize games that rely on Facebook-based social media platform to schedule court times.

The visibility that the social media platform provides exposes the various social networks to each other. Despite the exposure of these networks to each other, observation and communication across the social network is based on participation in the social media platform. The Facebook platform is primarily used for monitoring purposes.

\section{Vignette 2: Social media is a Relational Continuance Device}

Illustrative of social networks employing social media platforms as a relational continuance device we find that high school and secondary school social networks that use the platform. On these platforms the social networks communicate on a oneto-one or a one-to-many basis.

A university cohort social network that we identified through the interviews interacts through Facebook. Facebook use by the network is simultaneously private to the individual, while also being public. Parts of the social network like to flaunt behavior across the remainder of the social network while parts of the social network remain very subdued. Flaunting behavior is performed through sharing photos, videos and other types of provocative posts. Parts of the social network that flaunt, and the related commenting and posting behavior inform the remainder of the social network as to who is doing what and where. Facebook is used to observe the social network and occasionally touch them through the use of the commenting and the liking feature. The maintenance of relational linkages is dispersed, and social networks take place through the social media platform. Although the platform is not used for communication and coordination activities, it serves as a way to observe individuals in the network and as a way to maintain conversational and relational continuance through other modes of communication. In this vignette we saw the social media platform employed to maintain relational linkages across a specific network. These relational linkages are the weak ties that allow individuals to pick up lost threads of conversation.

\section{Vignette 3: Social media as an Observational Platform}

Some social networks use Facebook and LinkedIn as an observational platform rather than for direct and frequent interaction. Social networks employ these platforms to passively share information about their recent activities, and acts of direct communication or close coordination in the social network get transferred from the social media platforms to the more traditional modes of communication. For instance, informants have professional circles on LinkedIn and these professional circles include social networks of software developers, engineers and IT specialists. These social networks are referred to as a job opportunity networks. These social networks employ the social media platform to monitor the job opportunities and career opportunities that may be accessed through the network and platform. 
In the case of our informants, various social networks are accessed through social media platforms. The social media platform affords them the observational capability to peek into their social networks. Facebook is a platform through which interviewees observe the network at a distance and subsequently take direct communicative action. On the other hand, colleague-based social networks are within the LinkedIn social media platform. The informants for this vignette belong to social networks that employ social media technologies such as Facebook and LinkedIn.

\section{Vignette 4: Social media is an Emerging Social Infrastructure}

Social networks are diverse, fluid, geographically dispersed and are continually added to with new individuals. Fluid social networks are only one type of social network; social networks may also be highly stable and static. Static and stable social networks are typically family, extended family and high and secondary school networks.

The social networks described by our informants employ social media platforms to observe their networks but rely on traditional modes of communication for in-depth communication and coordination. However, specific networks encourage peripheral members of the network to communicate on social media platforms. While some networks treat Facebook as an observational platform they are aware of the need to engage further with their social network through the use of these platforms. They are aware of the increasing number of "friends" on these platforms and the need to interact with them. The social media platforms are an emergent social infrastructure for social networks in which they need to participate to potentially leverage their connections. For instance, the colleague social circle on LinkedIn is one that recognizes that such platforms provide access to a broad range of social networks that may be leveraged.

Social networks also use the social media platform to maintain relational continuance. Relational continuance in the network is important for participation in projects. For example, maintaining weak ties and awareness of the social network allows the network to function after the study abroad programs come to a close. In addition to maintaining relational continuance through these platforms, image projection on these platforms is also vital in maintaining certain social networks. The social network's continued engagement with the medium suggests that it recognizes the resources that are available through the social infrastructure embedded in these social media platforms.

\section{$5 \quad$ Analysis and Discussion}

The vignettes presented by the interviewees may be analyzed from two separate but complementary perspectives i.e. that of individual use and that of network-based adoption.

Our informants were quite capable of differentiating between the social media platforms they used and the social networks that they belonged to. They were also able to clearly articulate the adoption rationale, general use, benefits and barriers on behalf of 
the social network in their search for a suitable social media platform. We find in our research that the information and communication technologies adopted and used by individuals in the network in some instances were subsumed by the greater wishes of the social network. This is an interesting phenomenon where a higher level entity (the social network) is created by lower level entities (individuals and their relations), yet the higher level entity cannot be reduced to its parts (Andersen et al. 2000). Furthermore the higher-level entity has a direct causal effect on the lower level parts' behavior that created the higher-level entity in the first place, which is called downward causation. Here is means that the social network which is itself the result of many individuals and their mutual relations, exercises behavioral constraints on the very same individuals that constitute it.

The vignettes for social media as a communication and coordination medium and as a relational continuance device illustrate that the social network of the individual dictates adoption. This social network may be real or imagined on the part of the individual. Thus individuals that employ social media for these purposes employ the functional characteristics (Kietzmann, Hermkens, McCarthy, \& Silvestre, 2011) of conversation, groups, sharing and relationships to maintain and establish connections to their social networks. Vignette 1 illustrates this point as the grouping and conversation functionality of the Facebook social media technology was employed to participate in the social network of a high school camp. However vignette 1 also illustrates the individual use of the social media platform to establish and maintain presence on social media platforms. This presence can take many forms including the formation and maintenance of identities through the use of profiles or the monitoring of other identities and profiles. Consequently, the individual use of the social media platform takes place within the process of coordination within the social media platform.

Individual interviewees stated at several instances their conflicting use of social media vis-à-vis their adoption behavior. The quotes illustrate the manner in which individual use of the platform may not correspond with characteristics that the platform has despite these very characteristics driving adoption behavior.

"You have friends you want to talk to and you have friends you
don't want to talk to"....Udi
"I'm using Facebook to be social and show my life, there are
some people I don't care for looking at my photos, so, I have
been deleting a lot of friends"....Jannik

Vignette 2 describes the unique role that social media platforms play in maintaining social networks. These social networks are a result of dynamic and static ties between individuals but become a phenomenon of its own right. Naturally individuals belong to many such networks, some of which are very stable and established, and some of which are dynamic and much more fluid. Living in a digitalized age, digital natives employ information and communication technologies to establish, maintain and develop these social networks with social media being the most recent and principal of these technologies. As a relational continuance device the interviewees employed the relationship functional characteristic of the technology to create and maintain ties to other individuals within their social network. However their adoption and 
use of the social media platform was conditioned based on the existence of the social network on the platform.

However, the relationship continuance based on participation within social networks is moderated by the types of participants with whom individuals wish to interact. Individual use of the social media platform is moderated by the ease within which relationships are formed within the platforms. As the quotes illustrate, individuals condition their use based on the adoption by large parts of the network.

\section{“I don't use Facebook for my buddies, I use Facebook for my friends...."...Shu}

"Kammarat..its like a pal rather than a friend, a casual acquaintance. That's the word I use for people I just ignore and on Facebook I have a 300 (air quotes) "friends" but I don't want them to see what I am sharing with friends"...Shu

Vignette 3 illustrates the role of social media as observational platforms for dynamic social networks. Social media platforms serve as an observational platform where the individual can observe the changing nature of relationships and the formation of new ones through the use of the social media platform. As a communication and coordination medium, social media platforms offer the individual transparency. Communication in the social network on social media platforms is transparent to the entire global network. The boundaries of the network are porous and the addition of new members to the network may also be actively encouraged.

While individuals may adopt social media as an observation platform, the use of the media as such a platform does not necessarily imply they employ other communicative characteristics of the platform. Interviewees describe the use of the platform as observational and then the use of other media modes as a way to communicate and coordinate.

"I use Facebook for pictures mainly, vacation pictures, party pictures, videos, YouTube videos. I don't post on Facebook. I generally post once or twice a month. " "I don't talk to them on Facebook. I use it to see what is going on and then send them an e-mail."....Casper

\section{"I prefer the use of closed forums.".... Khaled}

Vignette 4 describes the social media platform as an emerging social infrastructure for the informal social networks. Relations are significantly altered when moved into the social media platform. On this platform, social networks participate by exposing some of the private into the public and as such expose the social networks to each other. One manner in which to manage this would be to maintain several social media platforms as infrastructure in which to manage networks. As we find, our informants' social networks do so by maintaining several networks in the social media platform, or by having more than one social media platforms as a basis for their social networks.

However individual use of the platform is constrained by the exposure the networks have to one another. Several interviewees exhibited concern that their relatives, 
family and other networks may get to see their behavior and participation with friends in other settings. Specifically individuals who employed the platform to communicate and interact with several social networks reflected this concern. Despite the adoption of the platform to maintain a social infrastructure by individuals, this participation constrained their individual use of the platform by constraining their use of characteristics and features.

\section{Conclusions}

In this paper we specifically examine the tension between individual use and networkbased adoption of social media platforms. The social network is an emergent entity of many individuals and their relations, yet it cannot be reduced to its constituent parts. Adoption perspectives on social media discuss the role that network effects and network behavior have on the adoption of the technology by the individual. The network lens drives us towards examining relations and relational structures that comprise groups and social networks. Consequently adoption behavior of individuals within social media platforms follows related network structures and relationships. However the adoption of a social media platform by an individual due to network behaviors constrains the use of the platform by the same individuals. These tensions have been referred to as socially mediated publicness (Baym et al. 2012) where collapsing contexts requires differing strategies on parts of individuals. We attempt to explore these strategies on the part of individuals and to explore how individuals manage or attempt to manage identities, relationships, and information in such social media platforms. While some individuals display behaviors of closing down communication and coordination on these platforms due to concerns of visibility (Marwick et al. 2012) others may choose strategies that are more suited to their concerns. Such strategies may take many forms that use affordances and characteristics of the technology to engage in posturing, power aggrandizement and reputation management (Lim et al. 2012). As the vignettes illustrate, the adoption of social media platforms have several facets, but each vignette illustrates the Janus-faced nature of the platform.

Vignette 1 illustrates the adoption of the technology as a communication and coordination medium but its affordances simultaneously constrain its use. Vignette 2 illustrates the adoption of the platform to maintain relationships but the use of the technology by individuals creates numerous relationships that are hard to distinguish and necessitate corrective behaviors in the part of individuals. Vignette 3 illustrates use of social media as an observational platform; however, the very act of observation is an act of communication that individuals are not comfortable with. And finally Vignette 4 illustrates the use of the platform as emerging social infrastructure; however, their use in such a form implies exposure of networks to one another.

The challenges of examining social media platforms with such an approach is that it requires researchers to focus on two fundamentally related processes, i.e. adoption and use. The primary question that emerges from the examination of this tension is; how do individuals make sense of conflicting goals within the adoption and use of 
social media platforms? While previous research may have examined social media adoption through individualistic lenses, they have included certain social network components in their analysis. Our focus has been to examine the individual use of the platform within the context of network-based adoption. We find that the tension between network-based adoption by individuals and individual use, points to interesting dynamics within the adoption and use of social media and the understanding of such dynamics may aid in understanding the success and failures of such technologies.

\section{References}

Ajzen, I.: From intentions to actions: A theory of planned behavior. Action-control: From Cognition to Behavior (11), 39 (1985)

Andersen, P.B., Emmeche, C., Finnemann, N.O.: Downward Causation: Minds, bodies and matter. Aarhus University Press (2000)

Arnold, M.: On the phenomenology of technology: the "Janus-faces" of mobile phones. Information and Organization 13(4), 231-256 (2003)

Attewell, P.: Technology diffusion and organizational learning: The case of business computing. Organization Science 3(1), 1-19 (1991)

Avital, M., Te'eni, D.: From generative fit to generative capacity: exploring an emerging dimension of information systems design and task performance. Information Systems Journal 19(4), 345-367 (2009)

Bagozzi, R.P.: The legacy of the technology acceptance model and a proposal for a paradigm shift. Journal of the Association for Information Systems 8(4), 244-254 (2007)

Bala, H., Venkatesh, V.: Assimilation of interorganizational business process standards. Information Systems Research 18(3), 340 (2007)

Baym, N.K., Boyd, D.: Socially Mediated Publicness: An Introduction. Journal of Broadcasting \& Electronic Media 56(3), 320-329 (2012)

Benbasat, I., Barki, H.: Quo vadis, TAM. Journal of the Association for Information Systems 8(4), 211-218 (2007)

Bhattacherjee, A.: Understanding information systems continuance: An expectationconfirmation model. MIS Quarterly 25(3), 351-370 (2001)

Boyd, D.M.: None of this is Real: Identity and Participation in Friendster. In: Karaganis, J. (ed.) Structures of Participation in Digital Culture, Social Science Research Council, United States of America (2007)

Davis, F.D.: Perceived usefulness, perceived ease of use, and user acceptance of information technology. MIS Quarterly 13(3), 319-340 (1989)

Davis, F.D., Bagozzi, R.P., Warshaw, P.R.: User acceptance of computer technology: A comparison of two theoretical models. Management Science 35(8), 982-1003 (1989)

Dishaw, M.T., Strong, D.M.: Extending the technology acceptance model with task-technology fit constructs. Information \& Management 36(1), 9-21 (1999)

Dutta, S.: What's Your Personal Social Media Strategy? Harvard Business Review 88(11), 127-130 (2010)

Fishbein, M., Ajzen, I.: Belief, attitude, intention, and behavior: An introduction to theory and research. Addison-Wesley, Reading (1975)

Gefen, D., Straub, D.W.: Gender differences in the perception and use of e-mail: An extension to the technology acceptance model. MIS Quarterly 21(4), 389-400 (1997) 
Granovetter, M.S.: The Strength of Weak Ties. The American Journal of Sociology 78(6), 1360-1380 (1973)

Hanna, R., Rohm, A., Cittenden, V.L.: We're all connected: The power of the social media ecosystem. Business Horizons (54), 265-273 (2011)

Hester, A.: Innovating with organizational wikis: factors facilitating adoption and diffusion of an effective collaborative knowledge management system, pp. 161-163. ACM (2008)

Hester, A.J., Scott, J.E.: A conceptual model of wiki technology diffusion, p. 32. IEEE Computer Society (2008)

Hsu, C.L., Lu, H.P.: Why do people play on-line games? An extended TAM with social influences and flow experience. Information \& Management 41(7), 853-868 (2004)

Iacovou, C.L., Benbasat, I., Dexter, A.S.: Electronic data interchange and small organizations: Adoption and impact of technology. MIS Quarterly, 465-485 (1995)

Jarvenpaa, S.L., Staples, D.S.: The use of collaborative electronic media for information sharing: an exploratory study of determinants. The Journal of Strategic Information Systems 9(2), 129-154 (2000)

Jones, B., Temperley, J., Lima, A.: Corporate reputation in the era of Web 2.0: the case of Primark. Journal of Marketing Management 25(9), 927-939 (2009)

Kietzmann, J.H., Hermkens, K., McCarthy, I.P., Silvestre, B.S.: Social media? Get serious! Understanding the functional building blocks of social media. Business Horizons (2011)

Kraut, R., Rice, R., Cool, C., Fish, R.: Varieties of Social Influence: The Role of Utility and Norms in the Success of a New Communication Medium. Organization Science 9(4), 437453 (1998)

Lee, A.S.: A scientific methodology for MIS case studies. MIS Quarterly 13(1), 33-50 (1989)

Lim, S.S., Vadrevu, S., Chan, Y.H., Basnyat, I.: Facework on Facebook: The Online Publicness of Juvenile Delinquents and Youths-at-Risk. Journal of Broadcasting \& Electronic Media 56(3), 346-361 (2012)

Limayem, M., Hirt, S.G.: Force of habit and information systems usage: Theory and initial validation. Journal of the Association for Information Systems 4, 65-97 (2003)

Lin, H.F.: Interorganizational and organizational determinants of planning effectiveness for Internet-based interorganizational systems. Information \& Management 43(4), 423-433 (2006)

Lyytinen, K., Damsgaard, J.: What's wrong with the diffusion of innovation theory. In: Proceedings of the IFIP TC8 WG8 (1), pp. 173-190 (2001a)

Lyytinen, K., Damsgaard, J.: What's wrong with the diffusion of innovation theory? Diffusing Software Products and Process Innovations, 173-190 (2001b)

Lyytinen, K., Damsgaard, J.: Inter-organizational information systems adoption - a configuration analysis approach. European Journal of Information Systems 18(1) (2011)

Mahler, A., Rogers, E.M.: The diffusion of interactive communication innovations and the critical mass: the adoption of telecommunications services by German banks. Telecommunications Policy 23(10-11), 719-740 (1999)

Malhotra, Y., Galletta, D.F.: Extending the technology acceptance model to account for social influence: theoretical bases and empirical validation, p. 14. IEEE (2002)

Markus, M.L.: Toward a "critical mass" theory of interactive media. Communication Research 14(5), 491 (1987)

Marwick, A., Ellison, N.B.: There Isn't Wifi in Heaven!" Negotiating Visibility on Facebook Memorial Pages. Journal of Broadcasting \& Electronic Media 56(3), 378-400 (2012)

McFarlan, F.W., McKenney, J.L., Pyburn, P.: The information archipelago-plotting a course. Harvard Business Review 61(1), 145 
Melville, N., Ramirez, R.: Information technology innovation diffusion: an information requirements paradigm. Information Systems Journal 18(3), 247-273 (2008)

Moore, G.C., Benhasat, I.: Development of an instrument to measure the perceptions of adopting an information technology innovation. Information Systems Research 2(3), 192-221 (1991)

Mustonen-Ollila, E., Lyytinen, K.: Why organizations adopt information system process innovations: a longitudinal study using Diffusion of Innovation theory. Information Systems Journal 13(3), 275-297 (2003)

O'Reilly, T.: What is web 2.0. Design patterns and business models for the next generation of software (30), 2005 (2005)

Parameswaran, M., Whinston, A.B.: Research issues in social computing. Journal of the Association for Information Systems 8(6), 336-350 (2007)

Peddibhotla, N.B., Subramani, M.R.: Contributing to public document repositories: A critical mass theory perspective. Organization Studies 28(3), 327 (2007)

Peng, G.: Critical Mass, Diffusion Channels, and Digital Divide. Journal of Computer Information Systems, 63-71 (2010)

Rogers, E.M.: Diffusion of innovations. Free Press (1995)

Salancik, G., Pfeffer, J.: A Social Information Processing Approach to Job Attitudes and Task Design. Administrative Science Quarterly 23(2), 224-253 (1978)

Sarker, S., Valacich, J.S.: Technology adoption by groups: A valence perspective. Journal of the Association for Information Systems 6(2), 37-71 (2005)

Sarker, S., Valacich, J.S.: An Alternative to Methodological Individualism: A NonReductionist Approach to Studying Technoogy Adoption by Groups. MIS Quarterly 34(4), 779-808 (2010)

Scott, S.V., Orlikowski, W.J.: 'Getting the truth': exploring the material grounds of institutional dynamics in social media (2009)

Sledgianowski, D., Kulviwat, S.: Using social network sites: the effects of playfulness, critical mass and trust in a hedonic context. Journal of Computer Information Systems 49(4), 74-83 (2009)

Straub, D., Keil, M., Brenner, W.: Testing the technology acceptance model across cultures: A three country study. Information \& Management 33(1), 1-11 (1997)

Tufekci, Z.: Grooming, Gossip, Facebook and MySpace. Information, Communication \& Society 11(4), 544-564 (2008)

Vannoy, S.A., Palvia, P.: The social influence model of technology adoption. Communications of the ACM 53(6), 149-153 (2010)

Venkatesh, V., Davis, F.D., Morris, M.G.: Dead or alive? The development, trajectory and future of technology adoption research. Journal of the Association for Information Systems 8(4), 267-286 (2007)

Venkatesh, V., Morris, M.G., Davis, G.B., Davis, F.D., DeLone, W.H., McLean, E.R., Jarvis, C.B., MacKenzie, S.B., Podsakoff, P.M., Chin, W.W.: User acceptance of information technology: Toward a unified view. Information \& Management 27(3), 425-478 (2003)

Yin, R.: Case Study Research: Design and Methods. Sage Publications, London (1989) 